\section{Acknowledgments}

We would like to express our thanks to Dr. Kunio Nakamura of Hokkaido University for his helpful suggestions. We are grateful to Dr. Taiichi Shibuya and Mr. Nobushige Kayama of Shinshu University for their helpful discussions and for reading the manuscript.

Nomenclature

$d \quad=$ diameter of pipe

$l=$ length of pipe

$\Delta p \quad=$ pressure drop

$\operatorname{Re} \quad=$ Reynolds number

$u \quad=$ average velocity of fluid $\lambda \quad=$ friction factor $\left(2 \Delta p d / \rho u^{2} l\right)$

Literature Cited

1) Imahara, K., K. Nakamura, T. Komatsu and T. Nakagawa: Nippon Kagaku Kaishi, 1551 (1983).

2) Lee, W. K., R. C. Vaseleski and A. B. Metzner: AIChE J., 20, 128 (1974).

3) Nakamura, K.: Kobunshi, 34, 86 (1985)

4) Virk, P. S.: AIChE J., 21, 625 (1975).

5) White, D. A.: Nature, 212, 277 (1966)

\title{
PREPARATION OF A NEW HYDROGEL AND POROUS GLASS COMPOSITE MEMBRANE
}

\author{
KATSUTO OTAKE, TOMOYA TSUJI, MIKIO KONNO \\ AND SHOZABURO SAITO
}

Department of Molecular Chemistry and Engineering, Tohoku University, Sendai 980

Key Words: Membrane Separation, Hydrogel, Porous Glass, Composite Membrane, Surface Modification

\section{Introduction}

Recently it has been learned that hydrogels, mixtures of crosslinked polymer networks having ionized groups and fluid media, undergo a discontinuous volume change with changes in environment such as temperature, $\mathrm{pH}$ or solvent composition. ${ }^{3)}$ This volume-phase transition is accompanied by changes in transport properties in the gels. Accordingly, the phase transition may be utilized in the creation of a new type of membrane with switching function. ${ }^{2}$ However, the gels' mechanical weakness must be overcome before they can be utilized in membranes. This may be attained by making a composite membrane of a gel and other materials. Recently, Nishi and Kotaka synthesized a gel membrane made of poly(oxyethylene)/poly(acrylic acid) interpenetrating polymer networks. ${ }^{1)}$ However, their method is restricted to polymer pairs which form an interpolymer complex.

In this paper, we report a new method for synthesizing composite membranes that should be applicable to various polymer-gels. The permeability of one new type of composite membrane of polymer-gel and

\footnotetext{
Received May 6,1988. Correspondence concerning this article should be addressed to S. Saito.
}

porous glass is also described.

\section{Experimental}

The inorganic porous substance used was porous glass plate (Asahi Glass Co. Ltd., average pore size $=$ $6900 \mathrm{~A}$ ), and the gel employed was crosslinked acrylamide (Am)/acrylic acid (AA) random copolymer.

Prior to the preparation of the composite membrane, the glass surface was modified by the following dehydration condensation reaction of $\mathrm{AA}$ and hydroxyl groups on the surface:

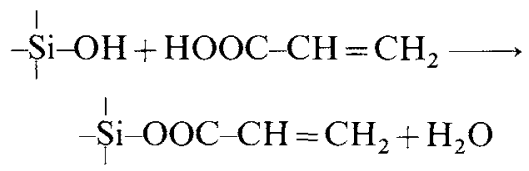

To support the gel on the porous glass, radical polymerization was employed. The two monomers, Am and AA, were used together with a crosslinking reagent, $N, N^{\prime}$-methylenebisacrylamide, and an initiation reagent, potassium persulfate. A prescribed amount of the chemicals was dissolved in water at a total monomer content of $10 \mathrm{wt} \%$ and an $\mathrm{Am} / \mathrm{AA}$ mole ratio of 4 to 1 . The porous glass was immersed in the aqueous monomer solution and saturated with it. Then polymerization was performed at $70^{\circ} \mathrm{C}$ for 
15 min. During polymerization, the gel formed was chemically fixed on the surface of the porous glass by reaction with the vinyl groups introduced by the surface modification.

All experiments were carried out at $25^{\circ} \mathrm{C}$ with the composite membrane prepared. The volume flux of aqueous acetone solutions was measured at various acetone concentrations. The pressure downstream of the membrane was maintained at atmospheric pressure and the pressure difference across the membrane ranged from $100 \mathrm{kPa}$ to $1500 \mathrm{kPa}$. The compositions of the feed and the permeate solution were measured by gas chromatography.

The swelling behavior of the gel is thought to affect membrane permeability. However, since it was difficult to observe the swelling behavior of the gels in the composite membrane, bulk gel of the same composition as the membrane was prepared. The equilibrium swelling volume of the bulk gel in the aqueous acetone solutions was measured at $25^{\circ} \mathrm{C}$, and the ratio of the swelling volume $V$ to the initial volume $V_{0}$ of the gel, the swelling ratio $V / V_{0}$, was determined.

Aqueous acetone solution containing poly(oxy-

Table 1.* Molecular weight and molecular weight distribution of POE

\begin{tabular}{lrrr}
\hline Reagent & \multicolumn{1}{c}{$M_{n}$} & $M_{w}$ & $M_{n} / M_{w}$ \\
\hline POE- 2000 & 2149 & 2192 & 1.019 \\
POE- 7000 & 7201 & 7283 & 1.011 \\
POE-20000 & 23549 & 24516 & 1.041 \\
\hline
\end{tabular}

* Measured by GPC.

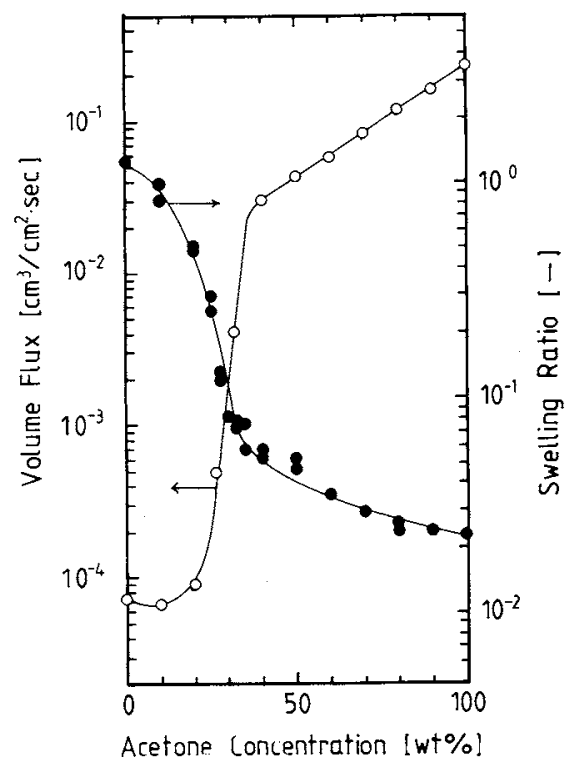

Fig. 1. Acetone concentration dependence of volume flux (open circles) of composite membrane at pressure difference $1500 \mathrm{kPa}$ and swelling ratio (closed circles) of bulk gel. Experiments were performed at $25^{\circ} \mathrm{C}$. ethylene)s (POE) of three molecular weights, POE$2000,-7000$ and $-20,000$, were used to examine the permeability further, also at $25^{\circ} \mathrm{C}$. Table 1 shows the properties of the POE. The compositions of the permeate solutions were measured by gel permeation chromatography (GPC) with refractive index unit.

\section{Results and Discussion}

Figure 1 shows the volume flux of the aqueous acetone solution through the membrane as a function of acetone concentration. In the same figure, the swelling ratio of the gel is also plotted. As the acetone concentration increased, the volume flux rapidly increased in the acetone concentration range around $30 \mathrm{wt} \%$. This concentration range corresponds to that in which the swelling ratio of the bulk gels changed considerably, from the swollen state to the shrunken state. Thus the composite membrane shows low volume flux in the swollen state and a high volume flux in the shrunken state.

Over the entire range of acetone concentrations, the volume flux was proportional to the pressure difference across the membrane. In addition, the composition of the permeate solution was the same as that of the feed solution.

Figures $2 \mathrm{a}$ and $\mathrm{b}$ show the experimental results of the GPC curves of the feed and permeate solution. In Fig. $2 \mathrm{a}$, when pure water was used as the solvent, POE- 20000 could not be detected in the permeate solution. Furthermore, peak heights of POE-7000 and 2000 were reduced by less than one-half.

Figure $2 \mathrm{~b}$ shows the results obtained with a $40 \mathrm{wt} \%$ acetone water solution solvent. The peak heights of the two POE solutions are about the same. This indicates that membrane permeability can be changed by solvent composition.

Figures 1 and 2 in this work exhibit a similarity to Nishi and Kotaka's observation on the dependence of the volume flux and the permeation characteristics on the swelling ratio. ${ }^{11}$
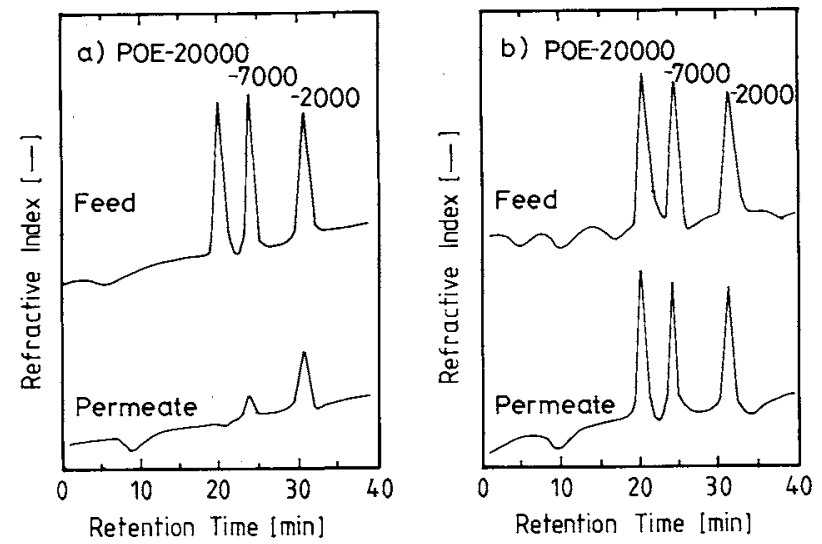

Fig. 2. GPC curves obtained with (a) pure water and (b) $40 \mathrm{wt} \%$ acetone water mixture at $25^{\circ} \mathrm{C}$ 
Although the present work describes only one kind of hydrogel supported on porous glass, the preparation method proposed here would be applicable to other hydrogels with inorganic porous substances. In addition, mass transfer experiments with gel may be performed much more simply with this new type of membrane.

\section{Literature Cited}

1) Nishi, S. and T. Kotaka: Macromolecules, 18, 1519 (1985); idem., ibid., 18, 978 (1985).

2) Osada, Y. and Y. Takeuchi: J. Polym. Sci., Polym. Lett. Ed., 19, 303 (1981).

3) Tanaka, T.: Phys. Rev. Lett., 40, 820 (1978). 